\title{
构建智能化实验开放共享平台的探索
}

吕银云, 潘荵, 翁玉华, 阮永红, 吴平平, 张来英, 彭淑女, 陈玉清, 张春艳,

董志强, 欧阳小清, 阮婵姿, 许振玲, 颜长明, 任艳平 ${ }^{*}$

厦门大学化学化工学院, 化学国家级实验教学示范中心(厦门大学), 福建 厦门 361005

摘要: 介绍了厦门大学化学国家级实验教学示范中心在构建智能化实验开放共享平台方面的一些探索经验, 阐述了 智能化实验开放共享平台的构建框架、管理系统、运行过程和运行成效, 以期为国内高校实验室开放管理提供可借 鉴经验。

关键词：智能化实验开放共享平台; 构建框架; 管理系统; 运行过程; 运行成效

中图分类号: G64; O6

\section{Exploration of Constructing the Intelligent Open Sharing Platform for Experiments}

Yinyun Lü, Rui Pan, Yuhua Weng, Yonghong Ruan, Pingping Wu, Laiying Zhang, Shunü Peng, Yuqing Chen, Chunyan Zhang, Zhiqiang Dong, Xiaoqing Ouyang, Chanzi Ruan, Zhenling Xu, Changming Yan, Yanping Ren *

National Demonstration Center for Experimental Chemistry Education (Xiamen University), College of Chemistry and Chemical Engineering, Xiamen University, Xiamen 361005, Fujian Province, China.

Abstract: This paper introduces practices and experiences of setting up the intelligent open sharing platform for experiments of the National Demonstration Center for Experimental Chemistry Education of Xiamen University, such as the construction framework, management system, operation process and operation effect, hoping to provide applicable guidance for the open management of domestic university laboratory.

Key Words: Intelligent open sharing platform for experiments; Construction framework; Management system; Operation process; Operation effect

厦门大学化学国家级实验教学示范中心(以下简称 “实验中心”)经过 10 多年的建设和发展, 其 硬件和软件资源日趋完备, 每学年承担着全校 10 个学院 3600 多名本科生的化学实验课教学任务, 实验中心在保障实验教学任务顺利完成之余, 如何充分利用实验中心的软硬件资源, 发挥其最大效 能，服务于广大师生，提高社会影响力，一直是实验中心考虑的问题。

进入新世纪以来, 我国高等教育进入内涵式发展时期, 建设创新型国家和人力资源强国对高层 次、拔尖创新人才的培养提出了更高的要求 ${ }^{[1,2]}$, 实验教学在本科化学教育和化学人才培养中起着基 础性的关键作用, 但有限的基础化学实验教学课时已经不能满足学生开展化学探究实验的需求, 学

收稿: 2020-03-19; 录用: 2020-04-27; 网络发表: 2020-05-06

*通讯作者, Email: ypren@xmu.edu.cn

基金资助：2018 年福建省本科高校教育教学改革项目(FBJG20180097); 2018 年厦门大学教学改革研究项目(JG20180105); 国家基础科学人才培 养基金项目(J1310024) 
生函需一个能在课外时间自由开展化学探究实验的平台; 党的十八大以来, 习近平总书记强调科学 普及与科技创新具有同等重要的位置 ${ }^{[3]}$, 作为国家级实验教学示范中心, 实验中心有责任、有义务 也有能力承担起向公众进行化学科学普及的重任, 开展化学科普工作义不容辞。

为了有效满足学生在课外开展化学探究实验的需求, 为了顺利有序开展化学科普活动, 同时将 实验中心的软硬件资源进行科学、有序开放共享, 构建智能化的实验开放共享平台势在必行。经过 一年多的探索和实践, 实验中心已经构建好一个相对完善的智能化实验开放共享平台(以下简称 “平 台” ) 。广大师生和校外人员可以通过平台查询实验中心现有药品试剂、仪器设备、预备实验项目等 信息, 以便更好地做好实验前的信息调研和准备工作; 学生可以在平台通过预约开展课外实验探索, 科研人员可以通过平台利用实验中心特有的仪器设备解决科研问题, 中小学生和社会公众可以通过 平台在实验中心开展化学科普实验, 领略和发现 “化学之美”。

智能化实验开放共享平台使得实验中心开放管理更加科学化、规范化、制度化和人性化, 取得 了良好的成效。下面简要谈谈智能化实验开放共享平台的构建框架、管理系统、运行过程和运行成 效，以期为国内高校实验室开放管理提供可借鉴经验。

\section{1 实验开放共享平台的构建框架}

实验开放共享平台的构建初衷为满足学生在实验教学课外之余开展化学探究实验的需求, 满足 中小学生和社会公众顺利开展化学科普活动的需求, 为了使相关人员能在平台快速查询相关资源并 预约开展实验, 实验开放共享平台的构建框架由 “信息查询” 和 “实验预约” 两个模块组成, 详细 的构建框架如图 1 所示。

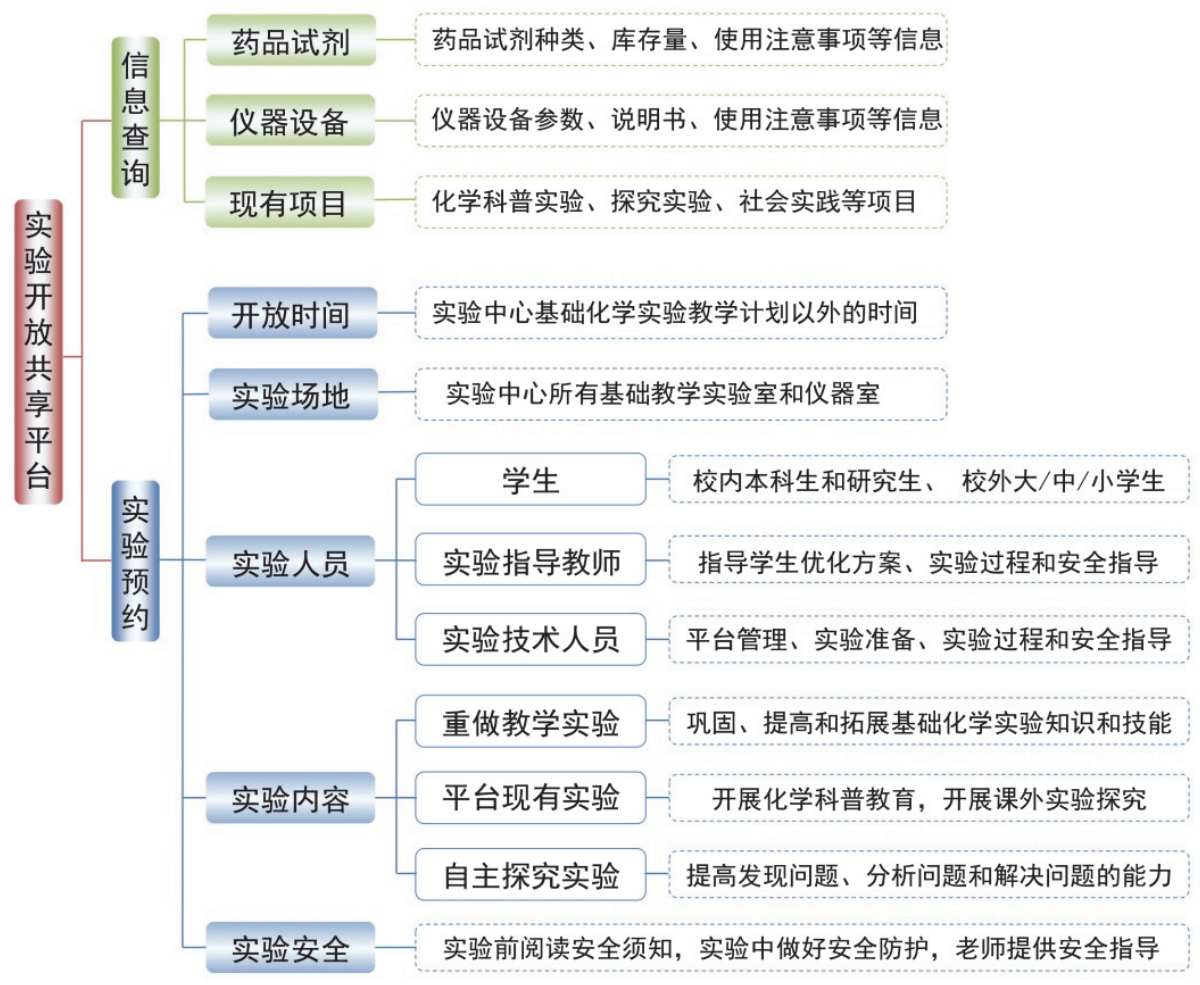

图 1 实验开放共享平台的构建框架

\section{1 信息查询模块}

通过信息查询模块可以查询实验中心现有药品试剂的种类、库存量和使用注意事项等, 可以查 
询实验中心现有仪器设备的参数、说明书和使用注意事项等, 可以查询实验中心预备的化学科普实 验、探究实验项目等。通过信息查询模块, 不仅能实现实验中心内部资源共享, 也能成为外界了解 实验中心资源的一个窗口, 同时也便于相关人员在了解实验中心资源的基础上制定可行的化学实验 方案, 再进行实验预约, 使得实验前的准备工作更加便捷、高效。

\section{2 实验预约模块}

实验预约模块包含 “开放时间” “实验场地” “实验人员” “实验内容” 和 “实验安全” ，学 生可以通过实验预约在实验中心重做基础教学实验、利用现有实验项目或自主设计实验项目开展科 普活动和化学探究实验, 也可以通过实验预约借用实验试剂、仪器等在实验中心以外开展科普活动 或化学探究实验, 满足校内外学生开展化学科普活动、提升课内化学实验知识和技能、开展化学探 究实验等各种需求。为了对学生进行全方位的化学实验安全教育, 学生在实验前必须了解化学实验 安全须知, 实验过程中在老师的指导下做好安全防护。

\section{2 实验开放共享平台的管理系统一实验预约系统}

实验开放共享平台有了初步的框架, 如何通过简便的管理工具实现预期功能呢? 早期, 实验中 心实验室对外开放过程中, 学生通过来来回回的邮件和实验中心老师沟通实验时间、实验地点和实 验方案, 过程繁琐、效率低。因此, 开发一个适用于化学实验室特点、交互信息强大的实验开放共 享平台管理系统对简化沟通过程、提高平台管理效率十分有必要。

经过半年的开发和调试, 实验开放共享平台管理系统一实验预约系统于 2018 年 12 月开发完成 并已上线, 系统采用 PHP 编程语言进行开发, 系统搭载在学校虚拟服务器运行, 由学校信息与网络 中心实时监测系统安全, 出现漏洞及时通知管理员进行修复, 从而保障系统安全。校内外人员可以 通过网站(http://kfpt.xmu.edu.cn)或微信( “XMU 化学实验中心” 公众号)进入系统进行信息查询和实 验预约, 实验预约系统各类信息的更新和预约申请审核由管理员在管理后台进行管理, 管理员由各 课程组指定专人担任。

实验开放共享平台管理系统一实验预约系统登录界面及下拉菜单界面如图 2 所示。校内外人员 直接点击菜单便可查询实验中心药品试剂、仪器设备和实验项目信息等, 并可下载相关说明进行深 入了解。

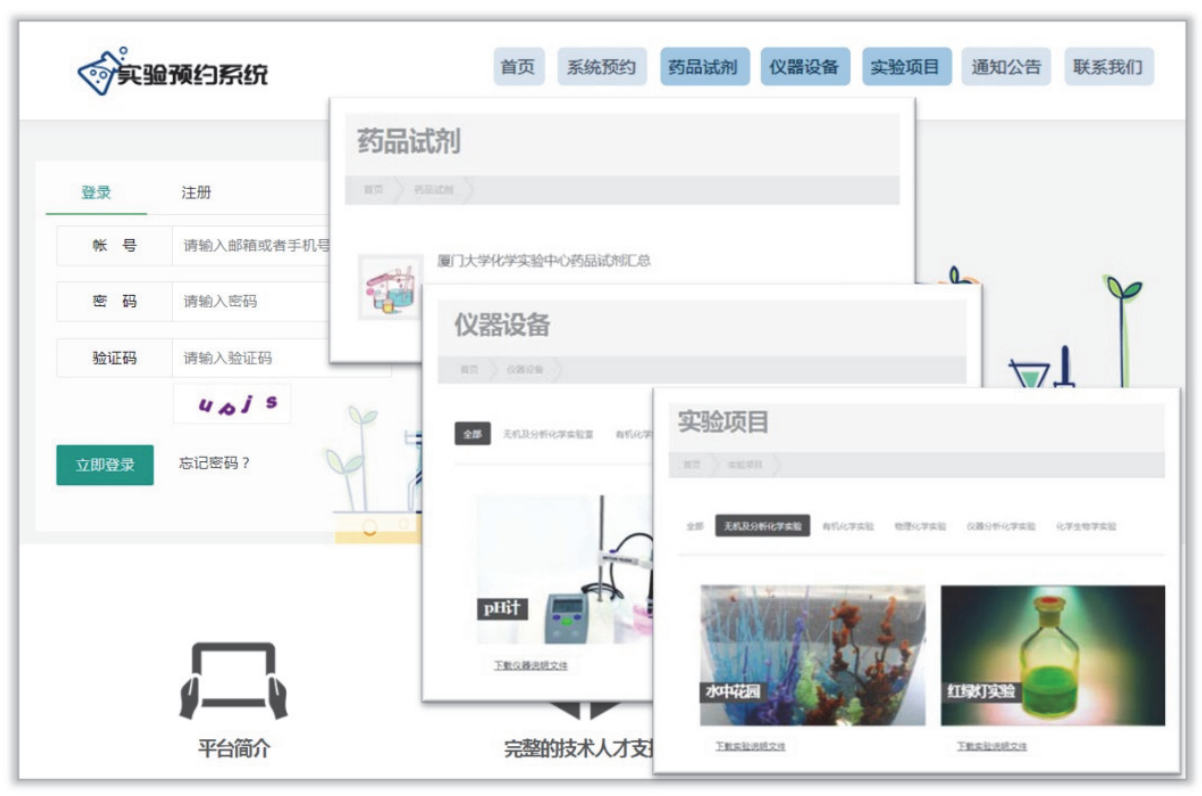

图 2 实验开放共享平台实验预约系统登录界面和下拉菜单 
在图 2 所示的界面注册账号并登录后的实验预约界面如图 3 所示, 实验预约系统根据实验开放 共享平台的构建框架设置了项目信息、联系人信息、实验人数、实验室选择、实验时间选择、实验 药品、实验仪器、备注、上传实验方案、安全须知等栏目, 学生不仅可以选填各个栏目信息进行实 验预约, 也能查询或下载实验中心的仪器设备和现有项目信息, 若只是借用实验试剂、仪器等在实 验中心以外开展科普活动或化学探究实验, 在备注栏进行说明即可。安全须知使得学生在实验前充 分了解实验过程中所要遵守的规章制度, 并让他们在实验时做好安全防护。

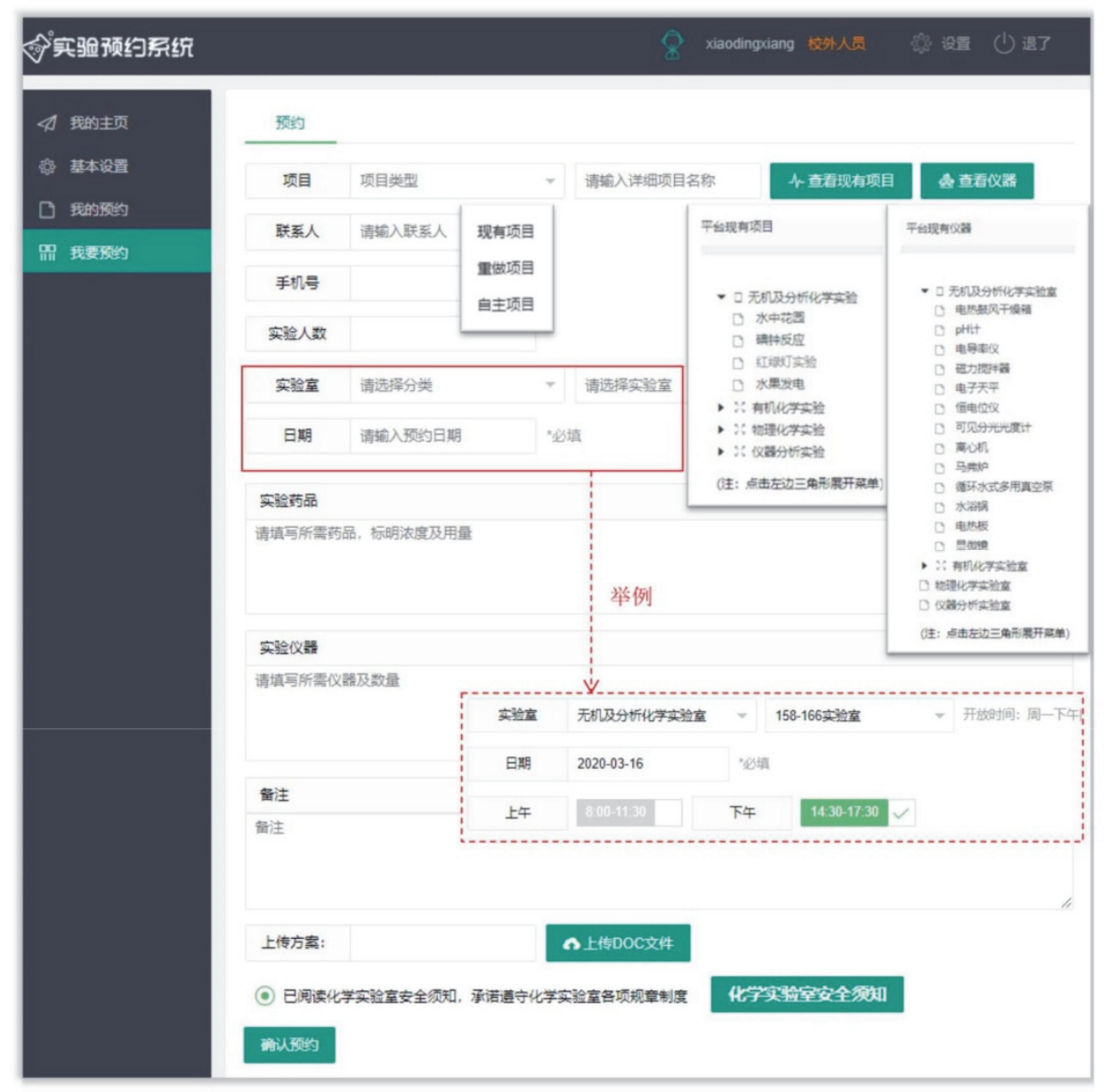

图 3 实验开放共享平台实验预约系统登录后界面和下拉菜单

\section{3 实验开放共享平台的运行过程}

实验开放共享平台的运行通过实验预约系统实现了从“信息查询一实验预约一管理员审核一实 验准备一开展实验一实验结束一再次实验预约” 的 “闭环” 管理, 使得学生在实验预约过程中或在 开展实验过程中的问题能有效得到解决, 进而顺利完成化学实验。具体运行过程如图 4 所示。

\section{1 信息查询}

在实验开放共享平台可以查询实验中心现有的药品试剂、仪器设备和现有项目, 便于实验者做 好实验预约前准备工作。

\section{2 实验预约}

学生通过注册实验预约系统账号, 登录后进行实验预约, 填写个人信息、实验时间、实验室、 实验药品、实验仪器和上传实验方案附件等, 并提交。 


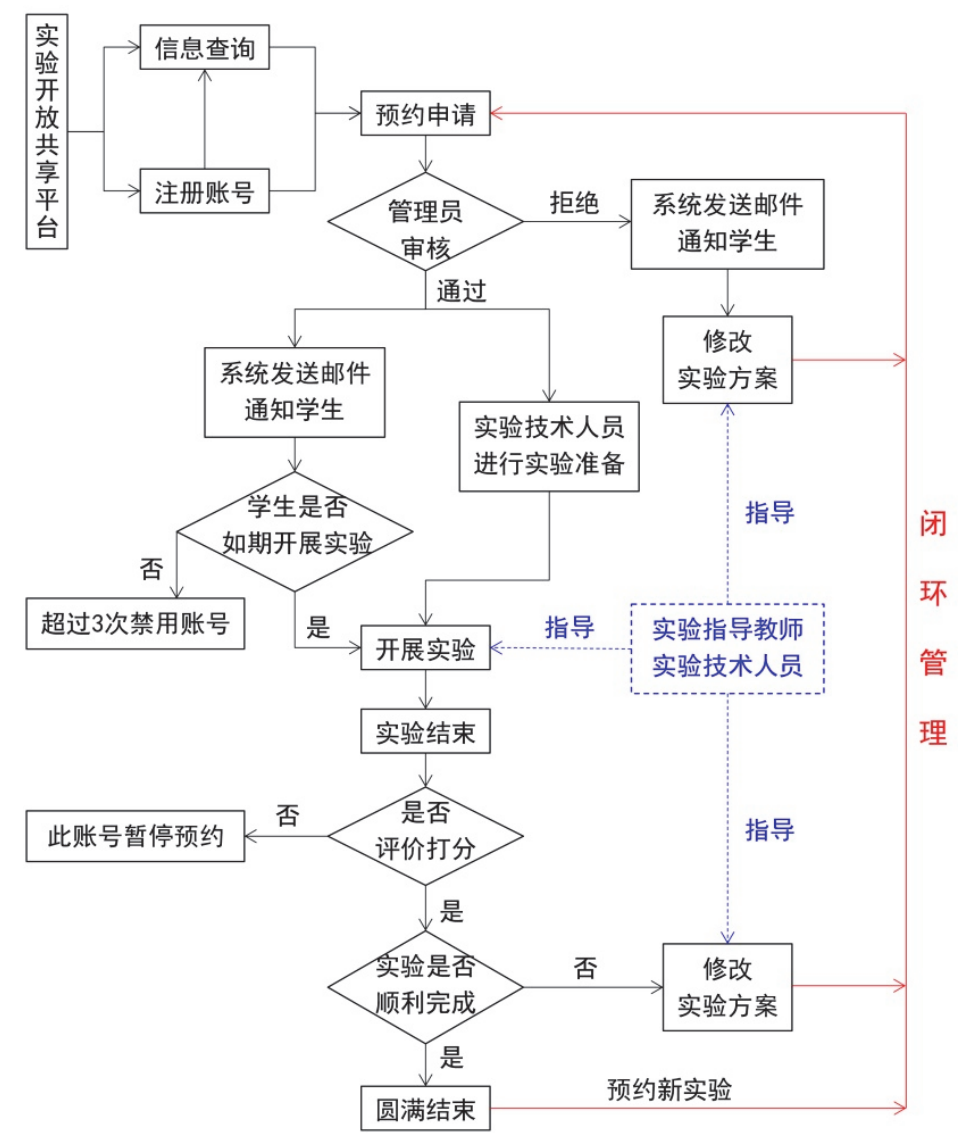

图 4 实验开放共享平台的运行过程

\section{3 管理员审核}

学生预约后, 管理员在管理后台对预约申请进行审核, 重点评估学生实验方案的可行性, 若方 案可行则 “通过申请” , 学生便可收到系统自动发送的实验预约成功的邮件; 若方案不可行则 “拒 绝” , 并填写拒绝理由, 学生会收到系统自动发送的实验预约失败的邮件并说明失败理由, 学生便 可在实验指导教师或实验技术人员的指导下修改实验方案再次预约。

\section{4 实验准备}

管理员通过学生的实验申请后，安排实验技术人员准备实验所需的药品试剂、仪器设备等。

\section{5 开展实验}

学生根据预约时间到达实验室开展实验, 实验指导教师或实验技术人员进行实验过程和安全指 导。若学生在未与老师沟通的情况下超过 3 次未按预定的时间开展实验, 则此学生的账号将被禁用 一个月。

\section{6 实验结束}

实验结束后, 学生对此次开展实验的情况进行评价打分, 可以对实验开放共享平台提出建设性 意见。若学生在预约时间内未完成实验或实验过程中遇到了新的问题, 可在实验指导教师或实验技 术人员的指导下修改实验方案, 再次预约, 继续完成实验。

\section{4 实验开放共享平台的运行成效}

实验开放共享平台依托实验中心运行, 实验中心的所有实验室在实验教学时间之外的空闲时间 全面对校内外学生开放, 实验开放共享平台为学生在课外开展化学探究实验提供了极大的便利, 也 
为校内外学生开展化学科普活动提供了交流的平台。尤其在 2018 年 12 月实验预约系统上线使用后, 使得实验开放共享平台的管理更具科学化、规范化、制度化和人性化, 取得了一定的成效。

\section{1 实验开放共享平台受益面广}

实验开放共享平台上线仅 1 年时间, 来自校内外的大、中、小学生们充分享受到了实验开放共 享平台带来的便捷。 2019 年度实验开放共享平台实验预约次数累计 195 次, 累计预约时长达 849 小 时, 共有 766 名学生通过平台在实验中心开展化学实验。实验人数中大学生占比 $46 \%$, 中学生占比 $48 \%$, 小学生占比 $6 \%$, 表明实验开放共享平台不仅满足了大学生开展化学探究实验的需求, 也满足 了校内外学生开展化学科普活动的需求(图 5a)。

\section{2 项目来源多元化}

学生通过平台在实验中心完成的实验项目可以是教学实验项目、现有实验项目和自主探究实验 项目, 实验项目来源呈现多元化。2019 年度通过平台在实验中心开展的 195 次实验中, 重做基础教 学实验项目占比 $6 \%$, 利用现有项目开展化学实验占比 $32 \%$, 自主设计实验项目并开展实验占比 $62 \%$ 。 表明实验开放共享平台充分满足了学生提升课内化学实验知识和技能、开展化学探究实验等需求(图 $5 b)$ 。

\section{3 实验内容多样化}

学生可根据自己的项目内容、所需试剂、仪器等具体情况在实验中心不同实验室开展化学实验, 实验内容呈现多样化。2019 年度通过平台在实验中心开展的 195 次实验中, 无机及分析化学实验占 比 $39 \%$, 物理化学实验占比 $35 \%$, 化学生物学实验占比 $23 \%$, 有机化学实验占比 $4 \%$, 充分说明了 各个年级的本科生都有开展化学探究实验的需求, 为学生们带来了极大的便利(图 5c)。

\section{(a)受益人群分析}

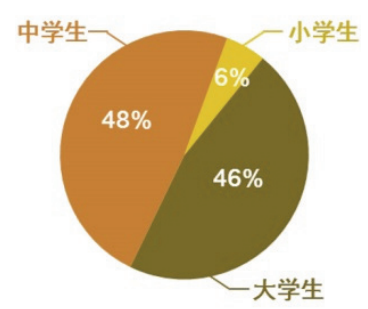

(b)项目来源分析

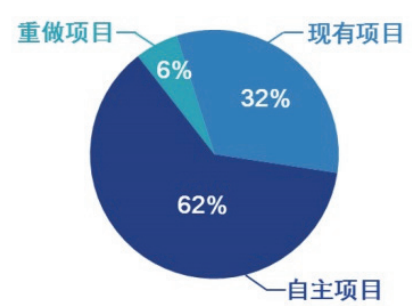

(c)实验内容分析

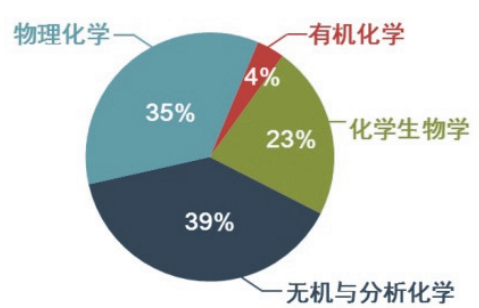

图 5 实验开放共享平台运行数据分析

学生通过平台在实验中心开展的实验项目来源多元化, 实验内容多样化, 使得实验方案五花八 门, 基本上是 “一人一案”, 这无疑给实验技术人员的实验准备工作增加了负担, 即便如此, 实验开 放共享平台却未收到任何差评, $73 \%$ 的学生评价 “极好”, $27 \%$ 的学生评价 “好”。学生评价: “预约 系统十分方便, 老师准备的很充分, 老师很耐心、非常认真负责、给了很多建议, 在实验室很顺利 地完成了实验, 谢谢实验室的老师们 .......” 这些好评离不开实验指导教师和实验技术人员的辛勤付 出, 也离不开实验开放共享平台科学化、规范化、制度化和人性化的设置和管理。

\section{5 结语}

厦门大学化学国家级实验教学示范中心构建的智能化实验开放共享平台经过 1 年多的运行实践, 平台的受益人数与日俱增, 通过平台在实验中心开展的实验项目来源多元化, 实验内容多样化; 充 分满足了校内外学生开展化学科普活动、提升课内化学实验知识和技能、开展化学探究实验等各种 需求; 使得实验中心的软硬件实现了全方位、立体式开放共享, 充分发挥了国家级实验教学示范中 心的社会服务职能。平台运行过程中得到了学生良好的反馈, 学生也提供了一些很好的建议, 如实 
验开放时间可以增多、希望预约时间从提前 7 天缩短为提前 3 天等, 我们也针对这些问题做了相应 改进，未来将继续完善实验开放共享平台的内容，提供更便捷、高效的服务。

参 考 文 献

[1] 管培俊. 中国高教研究, 2017, No. $12,17$.

[2] 卢连大. 现代教育科学, 2013, No. 1, 25.

[3] 习近平总书记对科协科普工作的重要指示. (2018-11-12) [2020-02-27]. https://www.sohu.com/a/274893122_100017525 